OPEN ACCESS

Edited by:

Fu Wang,

Xi'an Jiaotong University, China

Reviewed by:

Jingang Huang,

Sun Yat-sen Memorial Hospital, China

Chaoyun Pan,

Sun Yat-Sen University, China

${ }^{*}$ Correspondence:

Shuxin Song

shuxinsong@tom.com

Specialty section

This article was submitted to

Precision Medicine,

a section of the journal

Frontiers in Medicine

Received: 04 December 2020

Accepted: 11 January 2021

Published: 09 February 2021

Citation:

Sheng C, Chen Z, Lei J, Zhu J and

Song S (2021) Development and Multi-Data Set Verification of an RNA

Binding Protein Signature for

Prognosis Prediction in Glioma.

Front. Med. 8:637803.

doi: $10.3389 /$ fmed.2021.637803

\section{Development and Multi-Data Set Verification of an RNA Binding Protein Signature for Prognosis Prediction in Glioma}

\author{
Chunpeng Sheng, Zhihua Chen, Jianwei Lei, Jianming Zhu and Shuxin Song*
}

Department of Neurosurgery, The Second Affiliated Hospital of Nanchang University, Nanchang, China

Objective: Increasing evidence emphasizes the clinical implications of RNA binding proteins (RBPs) in cancers. This study aimed to develop a RBP signature for predicting prognosis in glioma.

Methods: Two glioma datasets as training $(n=693)$ and validation $(n=325)$ sets were retrieved from the CGGA database. In the training set, univariate Cox regression analysis was conducted to screen prognosis-related RBPs based on differentially expressed RBPs between WHO grade II and IV. A ten-RBP signature was then established. The predictive efficacy was evaluated by ROCs. The applicability was verified in the validation set. The pathways involving the risk scores were analyzed by SSGSEA. scRNA-seq was utilized for evaluating their expression in different glioma cell types. Moreover, their expression was externally validated between glioma and control samples.

Results: Based on 39 prognosis-related RBPs, a ten RBP signature was constructed. High risk score distinctly indicated a poorer prognosis than low risk score. AUCs were separately 0.838 and 0.822 in the training and validation sets, suggesting its well performance for prognosis prediction. Following adjustment of other clinicopathological characteristics, the signature was an independent risk factor. Various cancer-related pathways were significantly activated in samples with high risk score. The scRNA-seq identified that risk RBPs were mainly expressed in glioma malignant cells. Their high expression was also found in glioma than control samples.

Conclusion: This study developed a novel RBP signature for robustly predicting prognosis of glioma following multi-data set verification. These RBPs may affect the progression of glioma.

Keywords: RNA binding proteins, signature, glioma, prognosis, single cell RNA sequencing

\section{INTRODUCTION}

Glioma is the most frequently diagnosed primary brain malignancy, accounting for $70 \%$ of all brain malignancies (1). In line with the 2016 World Health Organization (WHO) classification, glioblastoma (GBM) is the most common histology type, which corresponds to WHO grade IV, with a median survival of $<2$ years and a 5 -year survival rate of $5 \%$ (2). 
The incidence of lower grade gliomas (LGG) WHO grade II is relatively lower and patients with grade II exhibit better clinical outcomes and more sensitive to therapies (3). At present, the diagnosis of glioma primarily depends on histopathology, imaging as well as molecular diagnosis (4-6). Nevertheless, because of complicacy and heterogeneity, traditionally diagnostic and therapeutic techniques exert side effects for the clinical outcomes of patients. To prolong the survival time of patients, it is of clinical importance for discovering novel accurate molecular biomarkers for prognosis prediction in glioma.

RBPs play major participants of the life cycle of mRNAs (7). One thousand five hundred forty-two RBP genes have been discovered in the human genome, corresponding to over $7.5 \%$ of protein-encoding genes (8). Totally, $\sim 50 \%$ of RBPs exert post-transcriptional mediated effects on gene expression (8). Abnormally expressed RBPs have been considered as drivers for cancers. They may be involved in regulating the progression and spread of cancers (9). Increasing evidence highlights the critical roles of RBPs in the malignant biological behaviors in gliomas (10). For example, RBP SRSF1 could regulate the cell cycle through stabilizing NEAT1 for glioma (11). RBP PCBP2 may modulate glioma growth via regulation of FHL3 (12). RBP SRSF3 regulates RNA alternative splicing, thereby inducing glioblastoma occurrence via influencing key biological processes (13). Nevertheless, their roles still require to be explored in depth via further functional studies. Recent studies suggest that several RBPs exhibit significant associations with clinical outcomes of glioma patients, such as SNRPN and IGF2BP3 (14). Hence, targeting RBPs appears to be promising strategies for the development of novel treatment against cancers. Moreover, it is of significance to understand the prognostic implications of RBPs in glioma. Herein, our findings developed a RBP signature for predicting the clinical outcomes of glioma patients. After validation, this signature exhibited a robust predictive efficacy.

\section{MATERIALS AND METHODS}

\section{Glioma Data Acquirement and Preprocessing}

Two glioma datasets ( $n=693$ or 325$)$ containing mRNA sequencing and corresponding clinical information were retrieved from the Chinese Glioma Genome Atlas (CGGA). A dataset ( $n=693$ ) was used as the training set and another ( $n$ $=325$ ) was utilized as the validation set. The batch effects were presented after integration of the two datasets.

\section{Differential Expression Analysis}

The mRNA expression profiles of RBPs were extracted from the training and validation sets. Differentially expressed RBPs were

Abbreviations: RBPs, RNA binding proteins; WHO, World Health Organization; LGG, lower grade gliomas; CGGA, Chinese Glioma Genome Atlas; FC, fold change; FDR, false discovery rate; ROCs, relative operating characteristic curves; KEGG, Kyoto Encyclopedia of Genes and Genomes; GO, Gene Ontology; ssGSEA, single sample Gene Set Enrichment Analysis; GSVA, Gene Set Variation Analysis; scRNA-seq, single cell RNA-sequencing; GEO, Gene Expression Omnibus; t-SNE, t-distributed stochastic neighbor embedding; GEPIA, Gene Expression Profiling Interactive Analysis; AUC, area under the curve. defined by comparing WHO grade II and IV samples via the edgeR package in $\mathrm{R}$ (15). The criteria were set as follows: |log fold change $(\mathrm{FC}) \mid \geq 0.58$ and false discovery rate $(\mathrm{FDR})>0.5$. Differentially expressed RBPs were visualized into volcano and heatmap plots.

\section{Univariate and Multivariate Cox Proportional Hazard Model}

Univariate cox regression analysis was conducted for differentially expressed RBPs in the training set. RBPs with hazard ratio $>1$ and $p<0.001$ were risk factors and those with hazard ratio $<1$ and $p<0.001$ were protective factors. RBPs with $p<0.001$ were chosen for multivariable cox regression. Totally, ten RBPs were filtered out for building up the predictive model. The risk score for each sample was calculated by combining the coefficient and the expression level of RBP. Afterwards, the patients were separated into high- and low-risk groups in line with the median values of the risk scores. Kaplan-Meier curves were constructed and the differences in survival time between groups were compared by log-rank test. Using the heatmap package in $\mathrm{R}$, the ten RBPs were displayed between the two groups. Relative operating characteristic curves (ROCs) were built for assessment of the predictive efficacy of the signature. Univariate and multivariate cox regression analysis was utilized to evaluate the associations between risk score as well as other clinical features and prognosis among glioma samples.

\section{Functional Enrichment Analysis}

Kyoto Encyclopedia of Genes and Genomes (KEGG) and Gene Ontology (GO) enrichment analyses were conducted for prognosis-related RBPs via the clusterProfiler package in R (16). Terms with adjusted $p<0.05$ were statistically significant.

\section{Single Sample Gene Set Enrichment Analysis (ssGSEA)}

Hallmark gene sets were obtained from the Molecular Signatures Database v7.2. ssGSEA was utilized to analyze the associations between risk scores and signaling pathways via the Gene Set Variation Analysis (GSVA) in R package (17). GSVA score in each pathway was calculated for a specific sample. The difference in a specific alteration in a pathway was compared between high and low risk groups via Wilcoxon rank-sum test.

\section{Construction and Evaluation of a Nomogram}

Based on the coefficients of the ten RBPs from the multivariate Cox regression analysis, the score was determined for each RBP. The total point was obtained by adding all scores of the RBPs. Through the function conversion relationship between the total point and the 1-, 2-, and 3-year survival probability, the predicted probability of the individual outcome event was calculated. The nomogram was conducted via the forestplot package in $\mathrm{R}$. Calibration curve was drawn for internal verification utilizing the rms package. The predicted 5-year survival was compared with the actual survival time. 


\section{Single Cell RNA-Sequencing (scRNA-seq) Data}

Three single cell RNA-seq datasets for glioma including GSE131928 Smart-seq2 (18), GSE131928 10X Genomics, GSE102130 datasets were retrieved from the Gene Expression Omnibus (GEO; https://www.ncbi.nlm.nih.gov/gds/). Following quality control, normalization and linear scaling analyses, cell cluster was presented via the Seurat package in R (19). Cell types were visualized by the $t$-distributed stochastic neighbor embedding ( $t-S N E)$. The expression levels of the ten RBPs were visualized in each cell type.

\section{Spearson Correlation Analysis}

The expression levels of IGF2BP3, RDM1, NSUN7, EXO1, APOBEC3F, FBXO17, FAM46A, ANG, ADARB2, and EIF4E1B were compared between glioma WHO grade II and IV samples. At the mRNA levels, Spearson correlation analysis was presented between these RBPs among the whole datasets.

\section{Gene Expression Profiling Interactive Analysis (GEPIA)}

Using the GEPIA database; (http://gepia2.cancer-pku.cn/\# index), the expression of RBPs was compared between tumor $(n=163)$ and normal $(n=207)$ specimens in the GBM dataset from TCGA and GTEX database. The cutoffs were set as $|\log 2 \mathrm{FC}|$ $>1$ and $p<0.01$.

\section{Reverse Transcription Quantitative Polymerase Chain Reaction (RT-qPCR)}

Ten pairs of glioma and normal tissue specimens were collected from The Second Affiliated Hospital of Nanchang university. All patients provided written informed content. This study was approved by the Ethics Committee of The Second Affiliated Hospital of Nanchang university (2020018). Total RNA was extracted from tissues utilizing TRIzol (Beyotime, Shanghai), which was reverse transcribed into cDNA. RT-qPCR was presented by the miScript SYBR Green PCR Kit (Applied Biosystems, USA). Table 1 listed the sequence information of primers. GAPDH was utilized as a control. The expression levels were calculated with the 2- $\Delta \Delta \mathrm{Ct}$ method. Using GraphPad Prism 7.0, the differences between tumor and normal groups were determined using student's $t$-test. $P<0.05$ indicated statistically significant.

\section{RESULTS}

\section{Screening Prognosis-Related RBPs for Glioma}

In this study, we compared the differences in expression of 1,487 RBPs between glioma WHO grade II and IV samples in the training set. With the criteria of $|\log \mathrm{FC}| \geq$ 0.58 and FDR $>0.5,40$ RBPs were abnormally expressed between glioma II and IV samples (Supplementary Table 1). Among them, 23 RBPs were up-regulated whereas 17 RBPs were down-regulated in glioma grade IV compared to II
TABLE 1 | The primer sequences for RT-qPCR.

\begin{tabular}{|c|c|}
\hline Gene name & Primer sequence $\left(5^{\prime}-3^{\prime}\right)$ \\
\hline ANG & $\begin{array}{l}\text { CTGGGCGTITGTTGTTGGTC (forward) } \\
\text { GGTTTGGCATCATAGTGCTGG (reverse) }\end{array}$ \\
\hline EXO1 & $\begin{array}{l}\text { TGAGGAAGTATAAAGGGCAGGT (forward) } \\
\text { AGTIITCAGCACAAGCAATAGC (reverse) }\end{array}$ \\
\hline FBXO17 & $\begin{array}{l}\text { CTGACCCGGTCCTTCAGTG (forward) } \\
\text { CTCCCGTACTGCTCAAAAGATAC (reverse) }\end{array}$ \\
\hline IGF2BP3 & $\begin{array}{l}\text { TATATCGGAAACCTCAGCGAGA (forward) } \\
\text { GGACCGAGTGCTCAACTTCT (reverse) }\end{array}$ \\
\hline NSUN7 & $\begin{array}{l}\text { GGACTCCGTITATGTCATGGC (forward) } \\
\text { CTCAGACTCGGACAAGGACC (reverse) }\end{array}$ \\
\hline
\end{tabular}

(Figure 1A). Heat maps visualized the expression patterns of these RBPs between glioma II and IV samples (Figure 1B). We explored prognosis-related RBPs in glioma via univariate cox regression analysis. Our data suggested that 39 RBPs were significantly associated with prognosis of glioma (Figure 1C). Among them, 22 RBPs were risk factors for glioma and 17 RBPs were protective factors for glioma. To further probe the biological functions of these prognosis-related RBPs, we carried out KEGG and GO enrichment analysis. Our data suggested that RNA transport, mRNA surveillance and RNA degradation pathways were distinctly enriched, as shown in Figure 1D. GO enrichment analysis results revealed that these RBPs were involved in mediating various key biological processes such as mRNA metabolic process, RNA splicing, mRNA processing, RNA transport and localization (Figures 1E,F). These data demonstrated that the prognosisrelated RBPs we selected could be involved in the progression of glioma.

\section{Establishment of an RBP Signature for Prognosis Prediction in Glioma}

Based on prognosis-related RBPs, in the training set, a tenRBP signature was established for glioma via multivariate cox regression analysis (Figure 2A). The risk score for each glioma was determined as follows: $0.772546974482237^{*}$ the expression levels of IGF2BP3 $+(-0.529440079138294) *$ the expression levels of RDM1 $+0.959791181375875 *$ the expression levels of NSUN7 $+1.28748170838841 *$ the expression levels of EXO1 $+(-0.92777652353892) *$ the expression levels of APOBEC3F $+0.746260894529048 *$ the expression levels of FBXO17 + $(-0.545584467299167) * \mathrm{FAM} 46 \mathrm{~A}+0.999495382483528 *$ the expression levels of ANG $+0.682420092015883 *$ the expression levels of ADARB2 $+(-0.565975323034877) *$ the expression levels of EIF4E1B. Among them, IGF2BP3 (HR: 2.165, 95\%CI: 1.547-3.030, $p<0.001$ ), NSUN7 (HR: 2.611, 95\%CI: 1.2265.561, $p=0.013$ ), EXO1 (HR: 3.624, 95\%CI: 1.978-3.030, $p<$ 0.001 ), FBXO17 (HR: 2.109, 95\%CI: 1.161-3.831, $p=0.014$ ), ANG (HR: 2.717, 95\%CI: $1.454-5.078, p=0.002)$ and ADARB2 (HR: $1.979,95 \% \mathrm{CI}: 1.308-2.992, p=0.001$ ) were negatively correlated with survival time for glioma patients. APOBEC3F (HR: 0.395, 95\%CI: 0.201-0.778, $p=0.007$ ) and EIF4E1B (HR: 


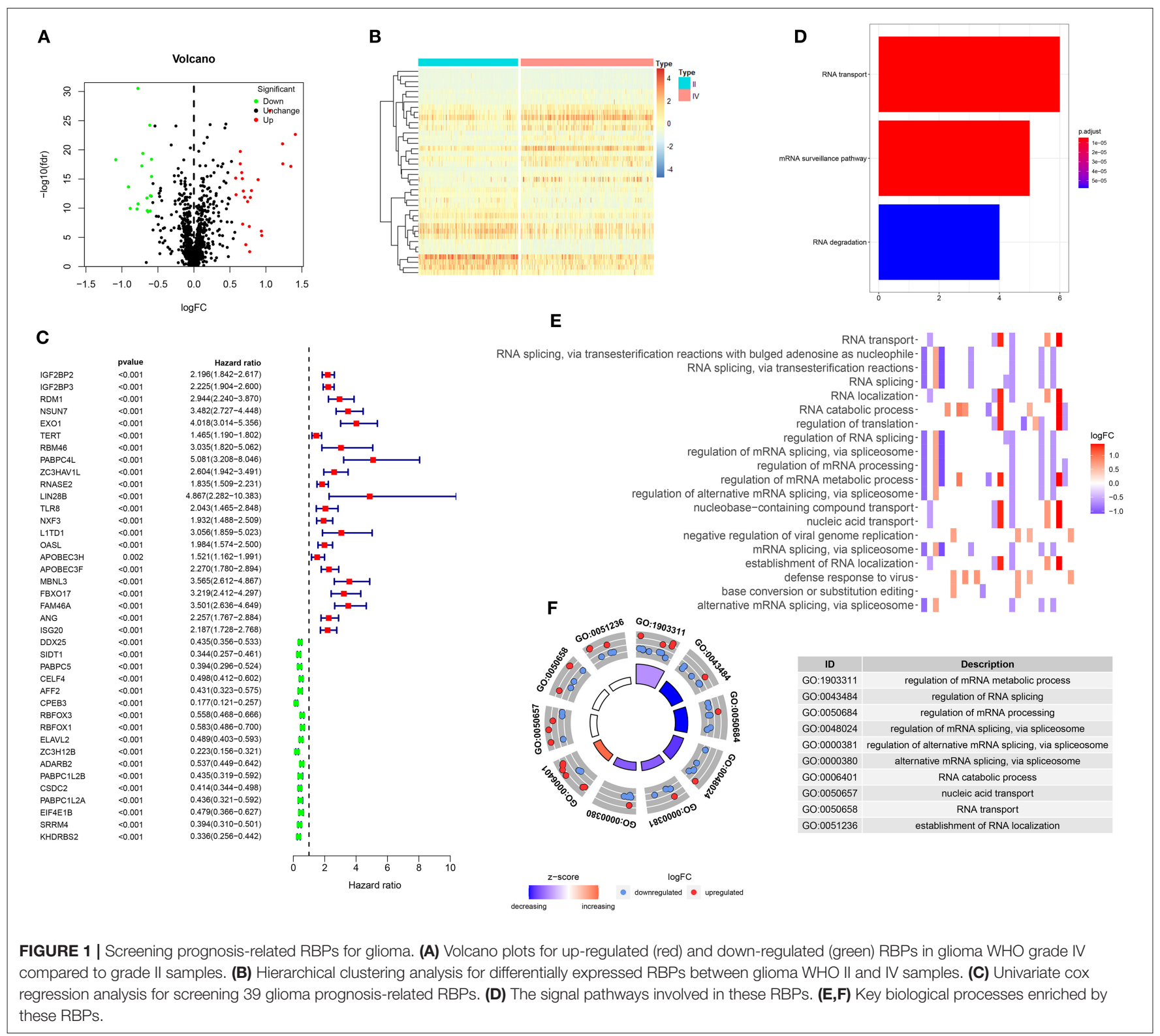

$0.56895 \%$ CI: $0.342-0.943, p=0.029)$ were positively associated with clinical outcomes in glioma patients. On the grounds of the median risk score, these patients were separated into high and low risk groups (Figure 2B). In comparison to the lowrisk group, the number of dead patients was higher in the high-risk group (Figure 2C). There were distinct differences in expression levels of these ten RBPs between the two subgroups (Figure 2D). The patients in the high-risk group exhibited an unfavorable prognosis compared to those in the low-risk group ( $p$ $<0.0001$; Figure 2E). The area under the curve (AUC) was 0.838, demonstrating that the signature possessed the well performance for prognosis prediction in glioma patients (Figure 2F). Based on the univariate cox regression analysis, PRS type (HR: 2.003, 95\%CI: 1.359-2.953, $p<0.001$ ), grade (HR: 3.008, 95\%CI:
2.297-3.938, $p<0.001$ ), age (HR: 3.797, 95\%CI: 2.444-5.900, $p<0.001$ ), chemotherapy status (HR: 1.911, 95\%CI: $1.151-$ 3.174, $p=0.012$ ) and risk score (HR: 1.294, 95\%CI: 1.229$1.363, p<0.001$ ) were risk factors for glioma (Figure 2G). We further assessed whether the signature could independently predict the clinical outcomes of glioma patients. Our multivariate cox regression analysis revealed the independency of the predictive efficacy of the signature (HR: 1.217 , 95\%CI: $1.143-$ 1.295, $p<0.001$; Figure 2H). Grade (HR: 2.702, 95\%CI: $1.948-$ 3.749, $p<0.001$ ) and age (HR: 1.975, 95\%CI: 1.249-3.123, $p=0.004)$ were both independently predictive of the clinical outcomes of glioma patients. Collectively, this signature could be robustly and accurately predictive of the clinical outcomes for glioma patients. 


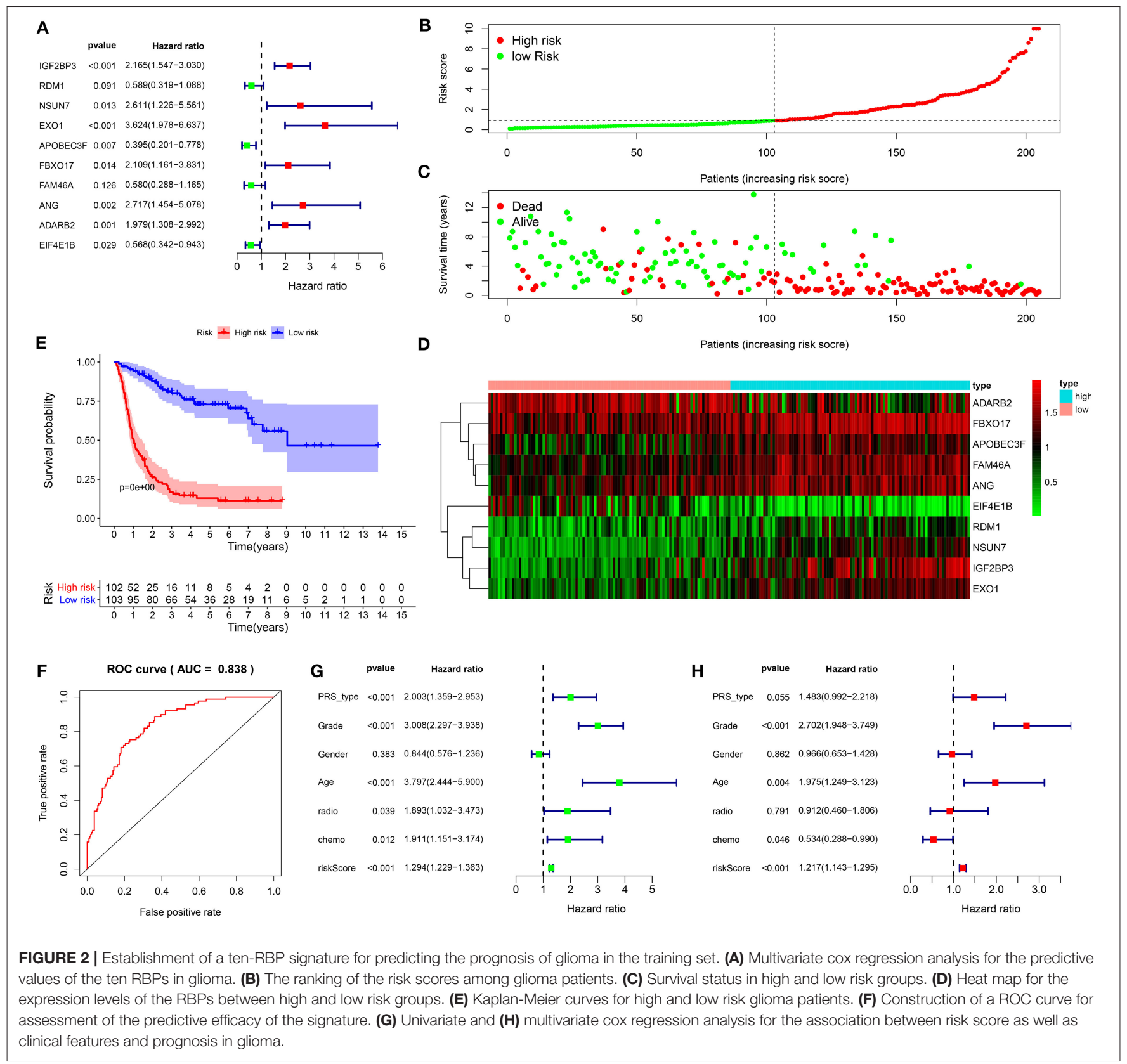

\section{Validation of the RBP Signature for Predicting Prognosis of Glioma}

We further verified the applicability of the signature for predicting prognosis of glioma in the validation set. Following the same method, patients were divided into high and low risk groups in accordance with the median value (Figure 3A). In the high-risk group, the number of dead patients was much higher than in the low-risk group (Figure 3B). In Figure 3C, these ten RBPs were abnormally expressed between the two subgroups. Patients in the high-risk group exhibited shorter survival time than those in the low-risk group $(p<0.0001$; Figure 3D). The high predictive value was confirmed by the
ROC curve (AUC $=0.822$; Figure 3E). PRS type (HR: 2.541, 95\%CI: 1.879-3.436, $p<0.001$ ), grade (HR: 2.868, 95\%CI: $2.360-3.486, p<0.001$ ), age (HR: 2.166, 95\%CI: 1.3863.386, $p<0.001$ ), chemotherapy (HR: 1.574, 95\%CI: $1.155-$ $2.145, p<0.004$ ), and risk score (HR: 1.298, 95\%CI: $1.248-$ 1.351, $p<0.001$ ) had negative correlations with prognosis of glioma patients (Figure 3F). Following the multivariate cox regression analysis, risk score (HR: 1.214, 95\%CI: $1.156-$ $1.274, p<0.001$ ), PRS type (HR: 1.958, 95\%CI: 1.4132.715, $p<0.001$ ), and grade (HR: 2.430, 95\%CI: $1.943-$ 3.040, $p<0.001)$ were independent risk factors for glioma (Figure 3G). 


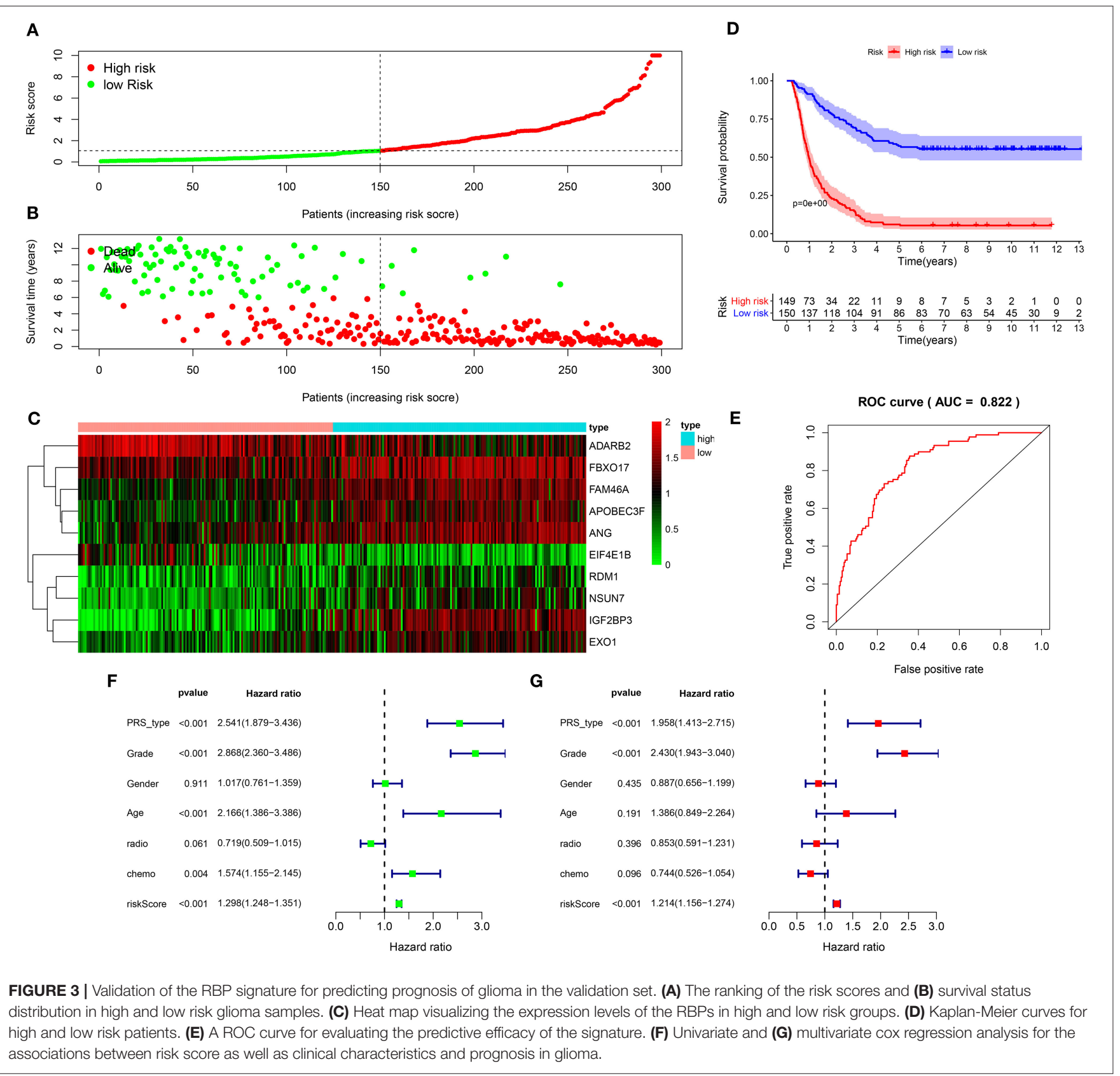

\section{ssGSEA Determines Signaling Pathways Involved in the Ten-RBP Signature}

GSVA score of a specific alteration in a pathway was calculated in each glioma sample. Our data suggested that the high-risk scores were significantly associated with hypoxia, TNFa signaling pathway via NF- $\kappa$ B, inflammatory response, mitotic spindle, PI3K-Akt-mTOR signaling pathway, Notch signaling pathway, interferon $\alpha$ response, interferon $\gamma$ response and glycolysis (all $p$ $<0.0001$; Figure 4). No difference in WNT $\beta$-Catenin signaling was found between high and low risk score groups. Above findings revealed that cancer-related pathways were activated in the glioma samples with high risk scores.

\section{Construction and Evaluation of a Nomogram for Prognosis Prediction of Glioma}

To personally calculate the survival rate of specific patient with glioma, a nomogram was established based on the ten RBPs in the training set. According to the influence levels of each RBP in the model on the clinical outcomes, each score of the RBP was assigned, and the total score was determined. The 1-, 2-, and 3-year survival probability of an individual patient was predicted based on the total scores (Figure 5A). We further evaluated the predictive efficacy of the nomogram in the validation set (Figure 5B). Calibration curves were depicted 


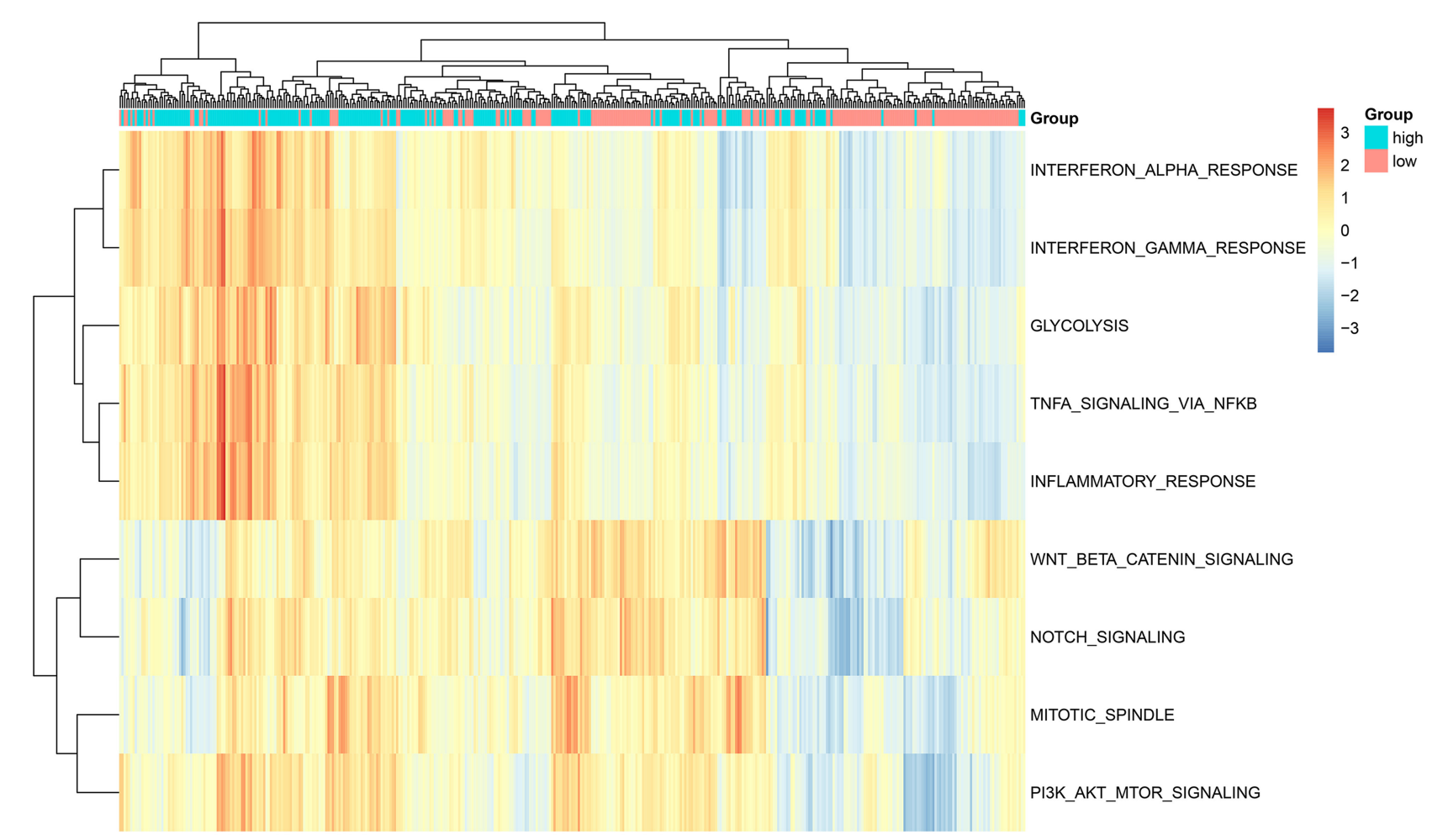

FIGURE 4 | ssGSEA determines the relationships between the ten-RBP signature and signaling pathways for glioma patients.

to compare the nomogram-predicted 5-year survival probability and actual survival time. Both in the training (Figure 5C) and validation (Figure 5D) sets, the nomogram exhibited the well performance for prediction of the 5-year survival time for glioma patients. Thus, the nomogram could be used for accurately assessing the survival probability for glioma patients.

\section{Single Cell RNA-seq Reveals the Expression of the Risk RBP Signatures in Glioma}

We deeply analyzed the expression levels of the risk RBP signatures in a single glioma cell in the three datasets. In the GSE131928 Smart-seq2 dataset, seven cell types were clustered, including AC-like malignant, CD8Tex, MES-like malignant, mon/macrophages, NPC-like malignant, OPC-like malignant and oligodendrocyte (Figure 6A). Consistent with our multivariate Cox regression analysis results, IGF2BP3, NSUN7, EXO1, and FBXO17 were highly expressed in AC, MES-, NPC-, and OPC-like malignant cells (Figures 6B-F). Furthermore, heat map visualized the expression patterns of EXO1 (Figure 6G), FBXO17 (Figure 6H), IGF2BP3 (Figure 6I), and NSUN7 (Figure 6J) in different cell types in the three datasets. Higher expression levels of the four RBPs were detected in the AC-, MES-, NPC- and OPC-like malignant cells, which could be related to poor prognosis for glioma patients.

\section{The Risk RBP Signatures Are Highly Expressed in Glioma Grade IV Than II}

We further compared the expression levels of the ten RBP signatures in glioma grade II and IV samples. The data showed that ADARB2 and EIF4E1B had lower expression levels in glioma grade IV than II samples (both $p<0.001$; Figure 7A). Most risk signatures exhibited higher expression levels of IGF2BP3, RDM1, NSUN7, EXO1, APOBEC3F, FBXO17, FAM46A, and ANG in grade IV than II. These results demonstrated that these risk signatures could affect the progression of glioma. Our study assessed whether these signatures could interact to promote tumor progression. The correlation analysis showed that, at the mRNA levels, there were obvious positive correlations between most of risk signatures (Figure 7B), such as RDM1 and EXO1 $(r$ $=0.53)$, ANG and FAM46A $(r=0.51)$, APOBEC3F and FBXO17 $(r=0.54)$, APOBEC3F and ANG $(r=0.7)$.

\section{The Risk RBP Signatures Are Highly Expressed in Glioma Than Controls}

The expression of risk RBP signatures was compared in glioma $(n=163)$ and control samples $(n=207)$ in TCGA-GTEx database. The results showed that ANG (Figure 8A), EXO1 (Figure 8B), FBXO17 (Figure 8C), IGF2BP3 (Figure 8D), and NSUN7 (Figure 8E) displayed distinctly higher expression levels in glioma compared to controls $(p<0.05)$. Furthermore, we validated their expression in 10 pairs of glioma and normal 


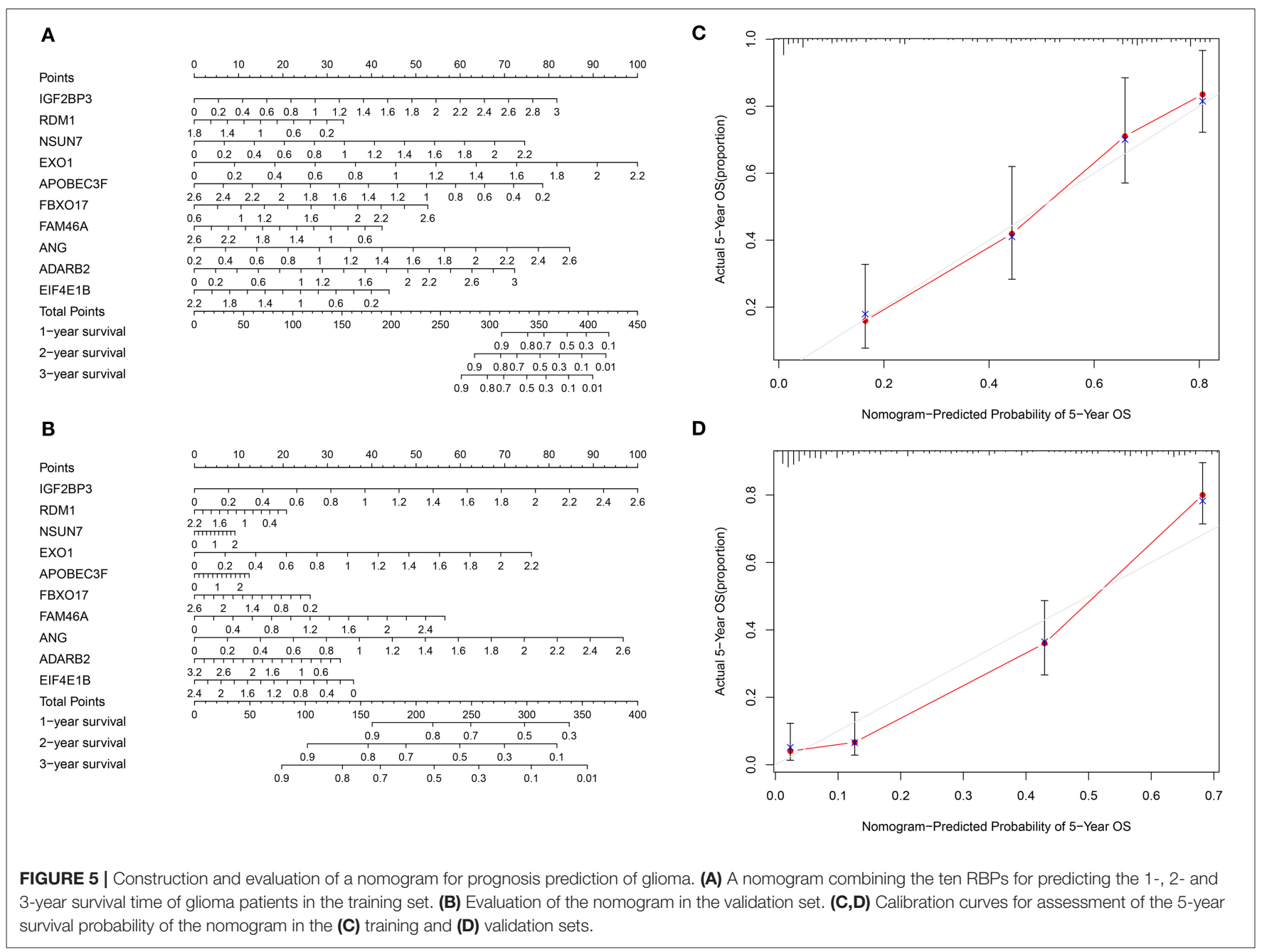

tissue specimens by RT-qPCR. Our data confirmed the upregulation of ANG (Figure 9A), EXO1 (Figure 9B), FBXO17 (Figure 9C), IGF2BP3 (Figure 9D), and NSUN7 (Figure 9E) in glioma than normal samples (all $p<0.0001$ ). These findings revealed that these risk signatures could participate in the progression of glioma.

\section{DISCUSSION}

Recent research demonstrates that several RBPs have closely correlations with the malignant behaviors of glioma (14). Nevertheless, the functions of most of the RBPs in glioma tumorigenesis remain unclear (20). In this study, based on prognosis-related RBPs, we established a ten-RBP signature for prognosis prediction in glioma. Following multi-dataset verification, this signature could robustly predict clinical outcomes for glioma patients.

Herein, 40 RBPs were differentially expressed between glioma WHO grade IV and II. Among them, 39 RBPs were correlated to prognosis of glioma, which might be involved in the malignancies of glioma. Hence, we probed the biological functions involving these RBPs. Our data suggested that these RBPs could posttranscriptionally mediate gene expression via the interaction with targeted RNAs, such as RNA transport, degradation, splicing and localization. Based on them, we developed a ten-RBP signature for glioma. The signature could be utilized for robustly predicting the prognosis of patients. Both in the training and validation sets, high risk score was predictive of poorer prognosis. AUCs were 0.838 and 0.822 in the two sets, respectively, suggesting the well performance. Following adjustment with other clinical features, the signature was an independent prognostic factor. As previous studies, several RBP signatures have been constructed for other cancers. For example, Li et al. built up a nine-RBP gene signature for lung squamous cell carcinoma prognosis (21). A six-RBP model constructed by Wu et al. demonstrated a well performance for predicting the clinical outcomes of bladder cancer (22). However, so far, there is still a lack of RBP-related signature for glioma. Our research fills this gap at some extents.

Our findings revealed that high-risk scores were markedly associated with various cancer-related pathways, like hypoxia, $\mathrm{TNF} \alpha$ signaling pathway via $\mathrm{NF}-\kappa \mathrm{B}$, inflammatory response, 


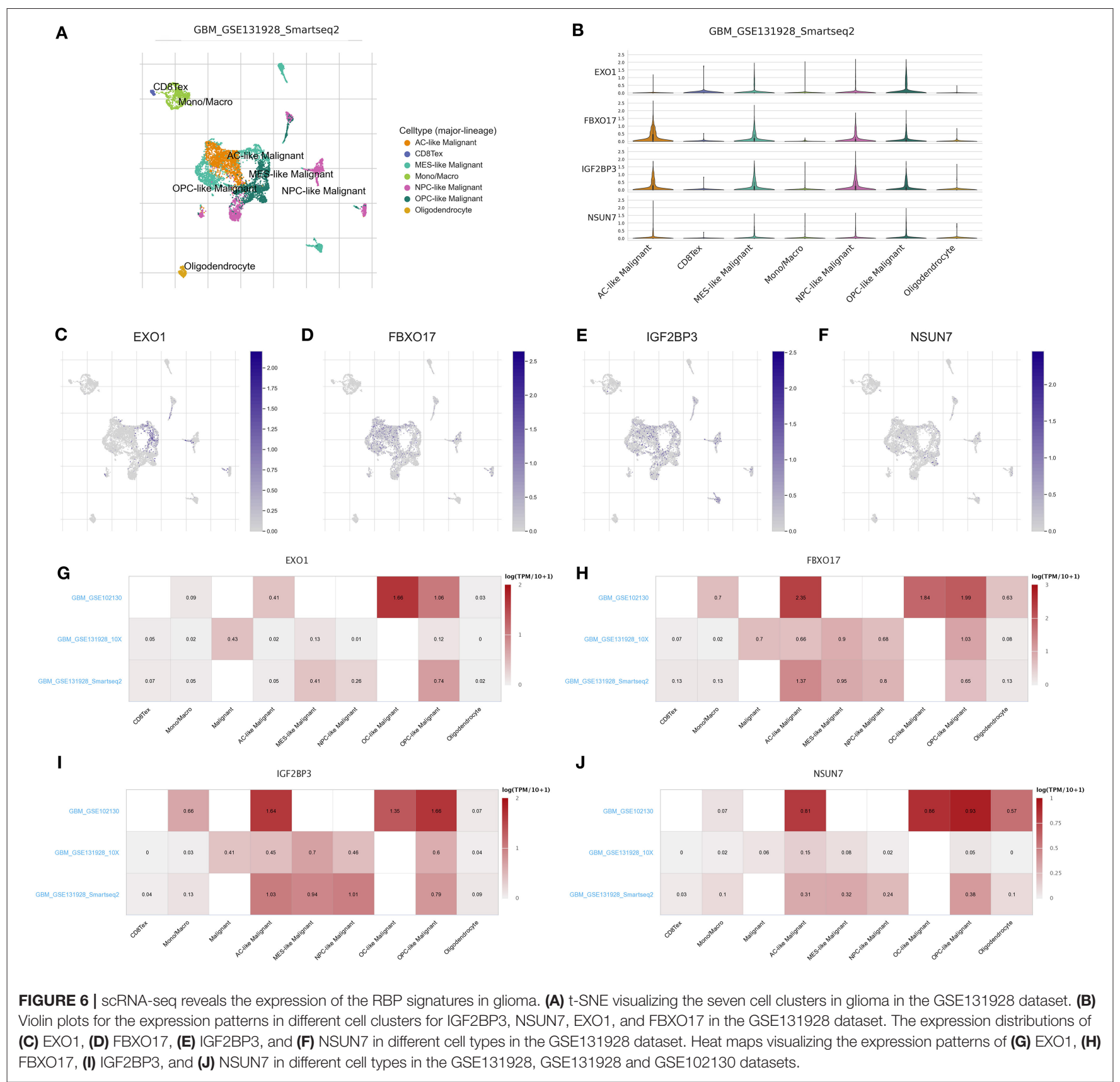

mitotic spindle, PI3K-Akt-mTOR, Notch, interferon $\alpha$ response, interferon $\gamma$ response and glycolysis pathways, indicating that cancer-related pathways were activated in glioma samples with high-risk scores. These RBPs could affect glioma progression via mediating above pathways. Previously, RBP ZEB1 facilitated hypoxia-mediated epithelial-mesenchymal transition in glioma cells (23). RBP MOV10 that bound to circ-DICER1 induced the proliferation, migration as well as tube formation in glioma cells via activation of PI3K/Akt pathway (24). RBP Musashil modulated the proliferative capacities of glioma cells via Notch and PI3K/Akt pathways.
Hence, activation of cancer-related pathways could be related to poor prognosis in high-risk score patients. To improve the clinical application potential of RBPs, we constructed a nomogram combining the ten RBPs for assessment of the 1-, 2-, and 3-year survival probability. This nomogram was validated in the validation set, indicating the high application values. After evaluation of calibration curves, the nomogrampredicted 5-year survival was highly consistent with the actual survival, suggesting that the nomogram possessed the potential as a scoring tool for predicting the clinical outcomes of patients. 
$\mathbf{A}$
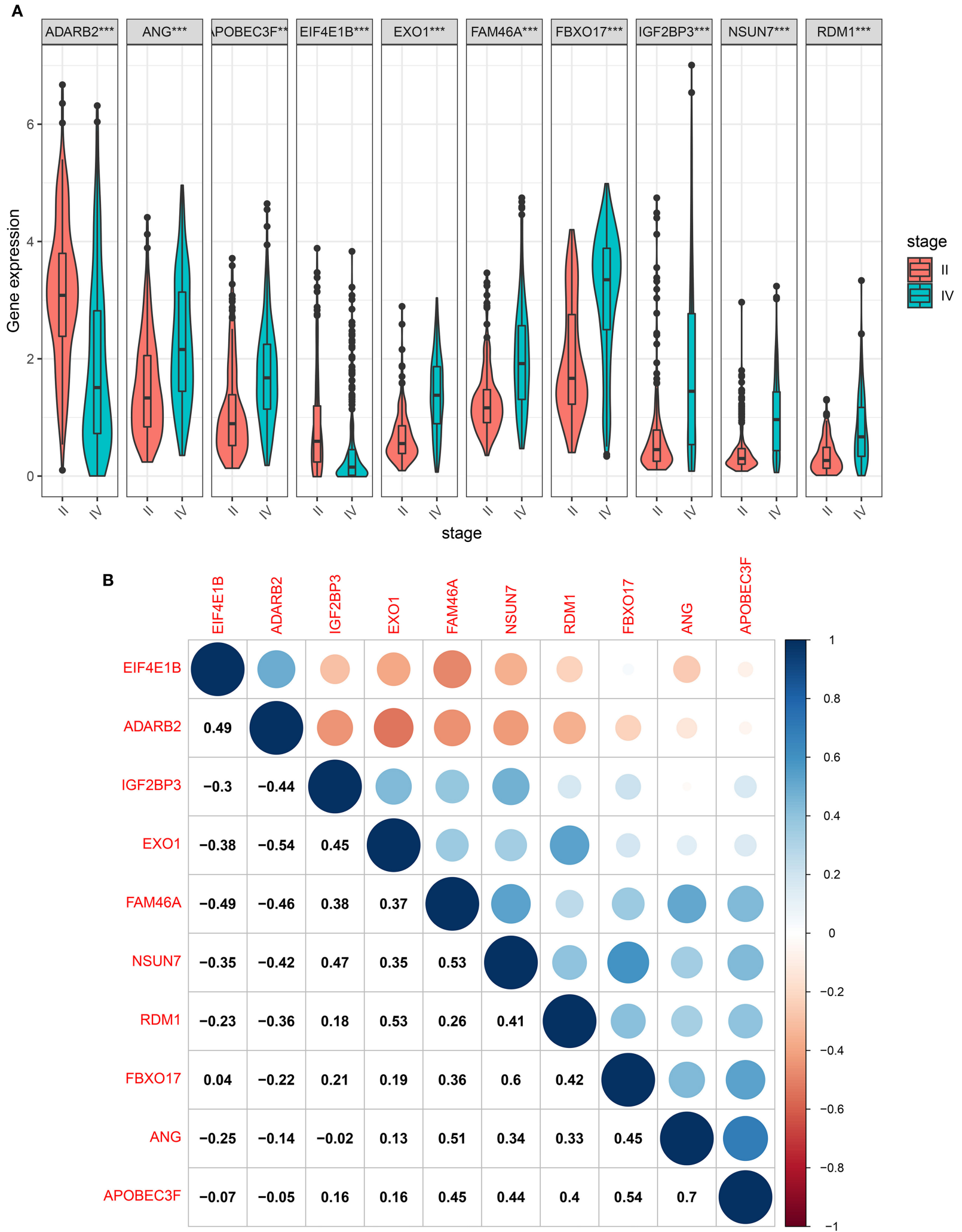

FIGURE 7 | The risk RBP signatures are highly expressed in glioma grade IV than II. (A) Box plots showing the expression levels of the ten RBP signatures in glioma grade IV and II samples. ${ }^{* *} p<0.001$. (B) The correlation between the ten RBP signatures at the mRNA levels. Blue: negative correlation; red: positive correlation. The size of the circle is proportional to the correlation coefficient. 


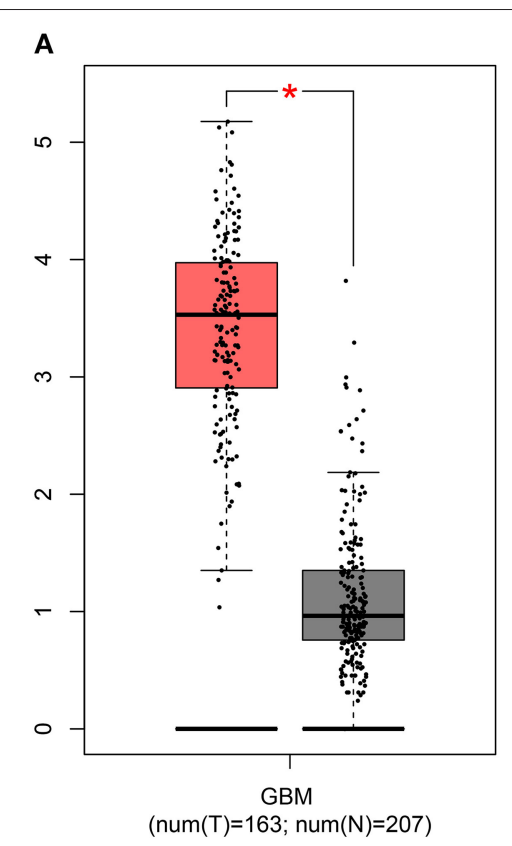

D

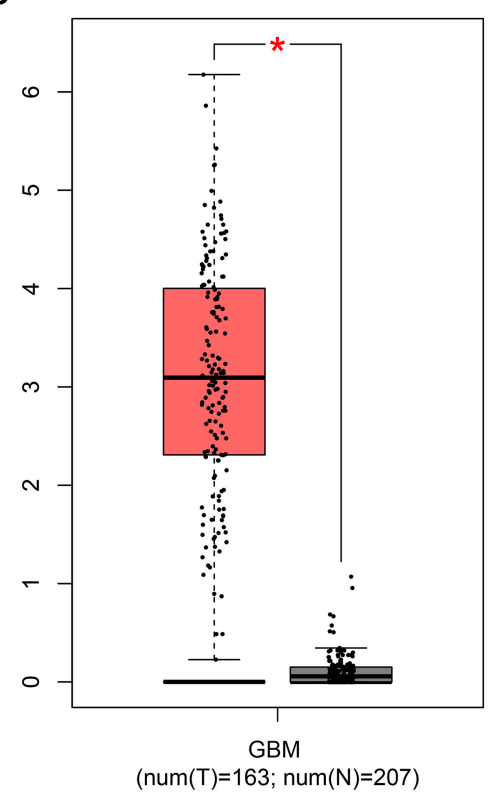

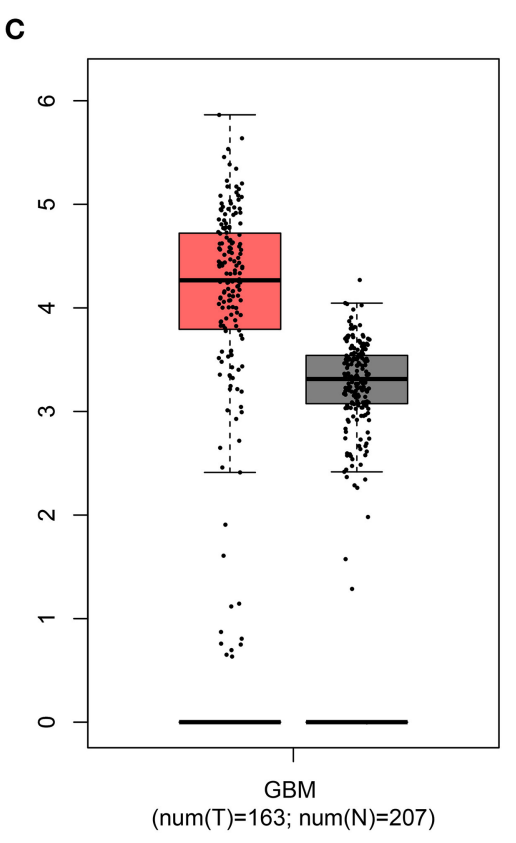

E

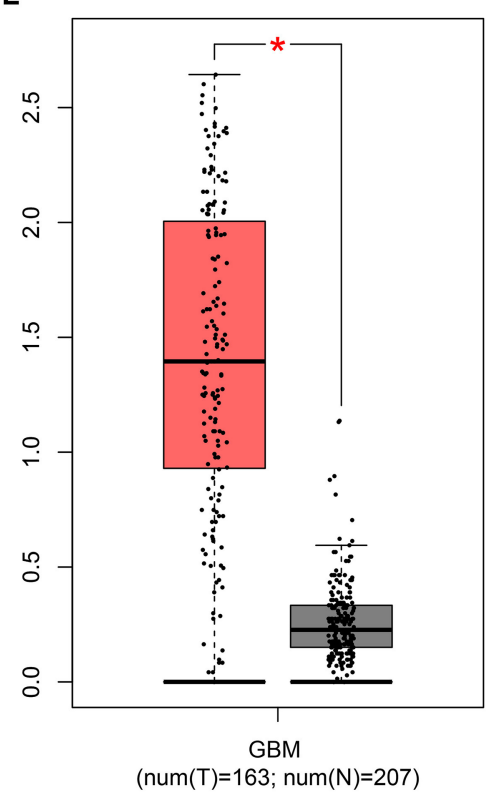

FIGURE 8 | GEPIA displays the highly expressed risk RBP signatures in glioma than controls. Box plots depicting the expression of (A) ANG, (B) EXO1, (C) FBXO17, (D) IGF2BP3, and (E) NSUN7 in glioma and control samples. ${ }^{*} p<0.01$.

We further explored why the high-risk scores indicated poor clinical outcomes. ScRNA-seq technology is widely used in basic scientific research and clinical research (25). Single cells have a place in many fields and are of great significance for the early diagnosis, tracking and individualized treatment of cancer $(26,27)$. The information displayed by traditional sequencing methods is also average information at the multi-cell level, while sequencing at the single-cell level can completely reflect the transcriptome status of different cells in the same cell group (28). Due to the high heterogeneity and complexity of glioma, the survival time of patients in the same pathological stage is completely different. It is of significance to present scRNAseq analysis for glioma. Herein, we detected the expression of the risk RBP signatures in different glioma cell types. Our data suggested that IGF2BP3, NSUN7, EXO1, and FBXO17 were highly expressed in AC-, MES-, NPC-, and OPC-like 


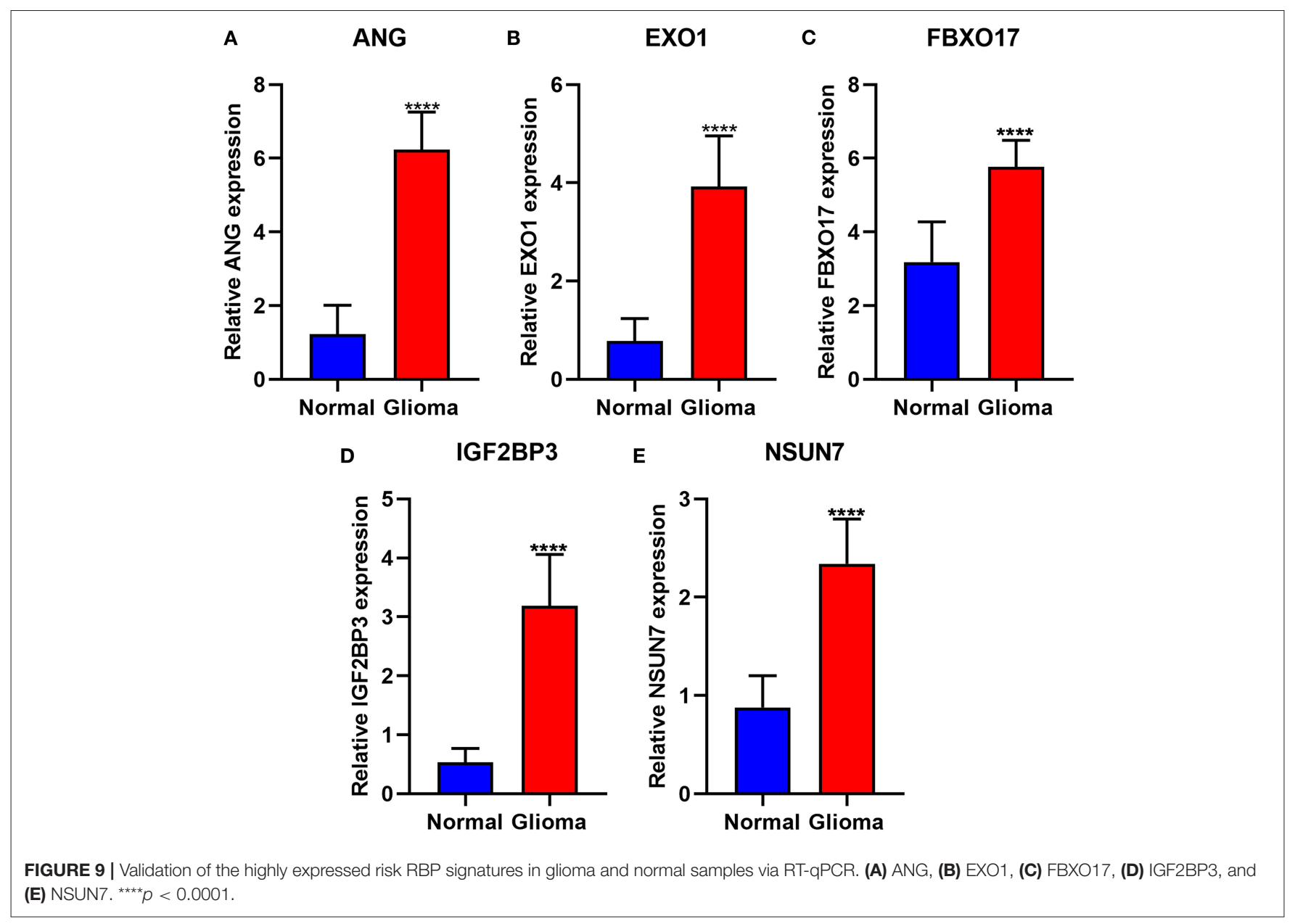

malignant cells, which could be related to poor prognosis for glioma patients. Furthermore, the four RBPs exhibited higher expression in WHO grade IV than II, indicating that they were associated with the malignancy of glioma. After validation by RT-qPCR, IGF2BP3, NSUN7, EXO1, and FBXO17 had higher expression levels in glioma than controls. As previous studies, IGF2BP3 facilitated viability and migration for glioma cells (29). NSUN7 is a risk factor for lower-grade glioma patients (30). EXO1 is associated with shorter survival time and hyposensitivity to temozolomide treatment in glioma (31). High FBXO17 expression could independently predict the clinical outcomes for high-grade glioma (32). Combining our results and previous research, these risk RBPs could accelerate the malignant behaviors of glioma.

In this study, a ten-RBP signature was constructed for glioma prognosis. This signature could independently predict the prognosis of patients with high accuracy and sensitivity. Combining RNA-seq and scRNA-seq analysis, we found that the four risk RBPs could contribute to the malignant behaviors of glioma. Their roles should be investigated by basic experiments and larger cohorts.

\section{CONCLUSION}

The 10-RBP gene signature exhibited a predictive efficacy for glioma prognosis under multi-data set verification. This signature may possess a promising application for clinical decision-making as well as individualized therapy.

\section{DATA AVAILABILITY STATEMENT}

The original contributions presented in the study are included in the article/Supplementary Material, further inquiries can be directed to the corresponding author/s.

\section{ETHICS STATEMENT}

The studies involving human participants were reviewed and approved by the Ethics Committee of The Second Affiliated Hospital of Nanchang university (2020018). The patients/participants provided their written informed consent to participate in this study. 


\section{AUTHOR CONTRIBUTIONS}

SS conceived and designed the study. CS and ZC conducted most of the experiments and data analysis, and wrote the manuscript. $\mathrm{JL}$ and JZ participated in collecting data and helped to draft the manuscript. All authors contributed to the article and approved the submitted version.

\section{REFERENCES}

1. Malta TM, de Souza CF, Sabedot TS, Silva TC, Mosella MS, Kalkanis SN, et al. Glioma CpG island methylator phenotype (G-CIMP): biological and clinical implications. Neuro Oncol. (2018) 20:608-20. doi: 10.1093/neuonc/nox183

2. Wang Z, Gao L, Guo X, Feng C, Lian W, Deng K, et al. Development and validation of a nomogram with an autophagy-related gene signature for predicting survival in patients with glioblastoma. Aging. (2019) 11:12246-69. doi: 10.18632 /aging.102566

3. Wang Z, Tang W, Yuan J, Qiang B, Han W, Peng X. Integrated analysis of RNA-binding proteins in glioma. Cancers. (2020) 12:892. doi: $10.3390 /$ cancers 12040892

4. Braunstein S, Raleigh D, Bindra R, Mueller S, Haas-Kogan D. Pediatric highgrade glioma: current molecular landscape and therapeutic approaches. $J$ Neurooncol. (2017) 134:541-9. doi: 10.1007/s11060-017-2393-0

5. Bush NA, Chang SM, Berger MS. Current and future strategies for treatment of glioma. Neurosurg Rev. (2017) 40:1-14. doi: 10.1007/s10143-016-0709-8

6. Booth TC, Williams M, Luis A, Cardoso J, Ashkan K, Shuaib H. Machine learning and glioma imaging biomarkers. Clin Radiol. (2020) 75:20-32. doi: 10.1016/j.crad.2019.07.001

7. Coppin L, Leclerc J, Vincent A, Porchet N, Pigny P. Messenger RNA life-cycle in cancer cells: emerging role of conventional and non-conventional RNAbinding proteins? Int J Mol Sci. (2018) 19:650. doi: 10.3390/ijms19030650

8. Gerstberger S, Hafner M, Tuschl T. A census of human RNA-binding proteins. Nat Rev Genet. (2014) 15:829-45. doi: 10.1038/nrg3813

9. Mohibi S, Chen X, Zhang J. Cancer the'RBP'eutics-RNA-binding proteins as therapeutic targets for cancer. Pharmacol Ther. (2019) 203:107390. doi: 10.1016/j.pharmthera.2019.07.001

10. Cao S, Zheng J, Liu X, Liu Y, Ruan X, Ma J, et al. FXR1 promotes the malignant biological behavior of glioma cells via stabilizing MIR17HG. J Exp Clin Cancer Res. (2019) 38:37. doi: 10.1186/s13046-018-0991-0

11. Zhou X, Li X, Yu L, Wang R, Hua D, Shi C, et al. The RNA-binding protein SRSF1 is a key cell cycle regulator via stabilizing NEAT1 in glioma. Int J Biochem Cell Biol. (2019) 113:75-86. doi: 10.1016/j.biocel.2019.06.003

12. Han W, Xin Z, Zhao Z, Bao W, Lin X, Yin B, et al. RNA-binding protein PCBP2 modulates glioma growth by regulating FHL3. J Clin Invest. (2013) 123:2103-18. doi: 10.1172/JCI61820

13. Song X, Wan X, Huang T, Zeng C, Sastry N, Wu B, et al. SRSF3regulated RNA alternative splicing promotes glioblastoma tumorigenicity by affecting multiple cellular processes. Cancer Res. (2019) 79:5288-301. doi: 10.1158/0008-5472.CAN-19-1504

14. Wang J, Qi J, Hou X. Systematically dissecting the function of RNAbinding proteins during glioma progression. Front Genet. (2019) 10:1394. doi: 10.3389/fgene.2019.01394

15. Robinson MD, McCarthy DJ, Smyth GK. edgeR: a Bioconductor package for differential expression analysis of digital gene expression data. Bioinformatics. (2010) 26:139-40. doi: 10.1093/bioinformatics/btp616

16. Yu G, Wang LG, Han Y, He QY. clusterProfiler: an R package for comparing biological themes among gene clusters. Omics. (2012) 16:284-7. doi: 10.1089/omi.2011.0118

17. Hänzelmann S, Castelo R, Guinney J. GSVA: gene set variation analysis for microarray and RNA-seq data. BMC Bioinformat. (2013) 14:7. doi: 10.1186/1471-2105-14-7

18. Hakes AE, Brand AH. Tailless/TLX reverts intermediate neural progenitors to stem cells driving tumourigenesis via repression of asense/ASCL1. Elife. (2020) 9:e53377. doi: 10.7554/eLife.53377.sa2

19. Butler A, Hoffman P, Smibert P, Papalexi E, Satija R. Integrating single-cell transcriptomic data across different conditions, technologies, and species. Nat Biotechnol. (2018) 36:411-20. doi: 10.1038/nbt.4096

\section{SUPPLEMENTARY MATERIAL}

The Supplementary Material for this article can be found online at: https://www.frontiersin.org/articles/10.3389/fmed. 2021.637803/full\#supplementary-material

Supplementary Table 1 | A list of 40 differentially expressed RBPs between glioma WHO grade II and IV samples.

20. Velasco MX, Kosti A, Guardia GDA, Santos MC, Tegge A, Qiao M, et al. Antagonism between the RNA-binding protein Musashil and miR-137 and its potential impact on neurogenesis and glioblastoma development. RNA. (2019) 25:768-82. doi: 10.1261/rna.069211.118

21. Li W, Li X, Gao LN, You CG. Integrated analysis of the functions and prognostic values of RNA binding proteins in lung squamous cell carcinoma. Front Genet. (2020) 11:185. doi: 10.3389/fgene.2020.00185

22. Wu Y, Liu Y, He A, Guan B, He S, Zhang C, et al. Identification of the sixRNA-binding protein signature for prognosis prediction in bladder cancer. Front Genet. (2020) 11:992. doi: 10.3389/fgene.2020.00992

23. Zhang S, Wang W, Liu G, Xie S, Li Q, Li Y, et al. Long noncoding RNA HOTTIP promotes hypoxia-induced epithelial-mesenchymal transition of malignant glioma by regulating the miR-101/ZEB1 axis. Biomed Pharmacother. (2017) 95:711-20. doi: 10.1016/j.biopha.2017.08.133

24. He Q, Zhao L, Liu X, Zheng J, Liu Y, Liu L, et al. MOV10 binding circDICER1 regulates the angiogenesis of glioma via miR-103a-3p/miR-3825p mediated ZIC4 expression change. J Exp Clin Cancer Res. (2019) 38:9. doi: 10.1186/s13046-018-0990-1

25. Ziegenhain C, Vieth B, Parekh S, Reinius B, Guillaumet-Adkins A, Smets M, et al. Comparative analysis of single-cell RNA sequencing methods. Mol Cell. (2017) 65:631-43.e634. doi: 10.1016/j.molcel.2017.01.023

26. Peng J, Sun BF, Chen CY, Zhou JY, Chen YS, Chen H, et al. Single-cell RNA-seq highlights intra-tumoral heterogeneity and malignant progression in pancreatic ductal adenocarcinoma. Cell Res. (2019) 29:725-38. doi: 10.1038/s41422-019-0195-y

27. Maynard A, McCoach CE, Rotow JK, Harris L, Haderk F, Kerr DL, et al. Therapy-induced evolution of human lung cancer revealed by single-cell RNA sequencing. Cell. (2020) 182:1232-51.e1222. doi: 10.1016/j.cell.2020. 07.017

28. Huang X, Liu S, Wu L, Jiang M, Hou Y. High throughput single cell RNA sequencing, bioinformatics analysis and applications. Adv Exp Med Biol. (2018) 1068:33-43. doi: 10.1007/978-981-13-0502-3_4

29. Jin P, Huang Y, Zhu P, Zou Y, Shao T, Wang O. CircRNA circHIPK3 serves as a prognostic marker to promote glioma progression by regulating miR654/IGF2BP3 signaling. Biochem Biophys Res Commun. (2018) 503:1570-4. doi: 10.1016/j.bbrc.2018.07.081

30. Sato K, Tahata K, Akimoto K. Five genes associated with survival in patients with lower-grade gliomas were identified by information-theoretical analysis. Anticancer Res. (2020) 40:2777-85. doi: 10.21873/anticanres. 14250

31. Wang Q, He Z, Chen Y. Comprehensive analysis reveals a 4-gene signature in predicting response to temozolomide in low-grade glioma patients. Cancer Control. (2019) 26:1073274819855118. doi: 10.1177/10732748198 55118

32. Du D, Yuan J, Ma W, Ning J, Weinstein JN, Yuan X, et al. Clinical significance of FBXO17 gene expression in high-grade glioma. BMC Cancer. (2018) 18:773. doi: 10.1186/s12885-018-4680-3

Conflict of Interest: The authors declare that the research was conducted in the absence of any commercial or financial relationships that could be construed as a potential conflict of interest.

Copyright (C) 2021 Sheng, Chen, Lei, Zhu and Song. This is an open-access article distributed under the terms of the Creative Commons Attribution License (CC BY). The use, distribution or reproduction in other forums is permitted, provided the original author(s) and the copyright owner(s) are credited and that the original publication in this journal is cited, in accordance with accepted academic practice. No use, distribution or reproduction is permitted which does not comply with these terms. 